\title{
Less is More: Video-Assisted Thoracic Surgery (VATS) vs. Open Thoracotomy (OT) in the Management of Resectable Lung Cancer
}

Shagufta Shaheen ${ }^{1}$, Salman Otoukesh ${ }^{1 *}$, Brice Jabo ${ }^{2}$, Manmeet Kaur ${ }^{1}$, Nicole Wheeler ${ }^{1}$, Saied Mirshahidi ${ }^{3}$, Salman Zaheer ${ }^{1}$ and Hamid R Mirshahidi ${ }^{1}$

${ }^{1}$ Loma Linda University Medical Center, Loma Linda, USA

${ }^{2}$ Loma Linda University School of Public Health, Loma Linda, USA

${ }^{3}$ Loma Linda University Cancer Center, Biospecimen Laboratory, Loma Linda, USA

\begin{abstract}
Background: Video-Assisted Thoracoscopic Surgery (VATS) has become the recommended approach for the treatment of resectable lung cancer. However, no large randomized clinical trial has been conducted formally comparing surgical resections completed by VATS to those done by Open Thoracotomy (OT) in low volume centers. The current study sought to assess differences in Recurrence-Free Survival (RFS), Overall Survival (OS), positive margins and postoperative Length of Stay (LOS) between VATS and OT lobectomies in our center.
\end{abstract}

Method: A single institution retrospective chart review from May 2005 through May 2015 was conducted. All patients diagnosed with stage I through III lung cancer who received surgical resection were selected. Patient and tumor characteristics recorded included age at diagnosis, sex, tobacco use, tumor location (side and lobe), stage, size and receipt of chemotherapy or radiotherapy. Chi-square and Wilcoxon-Mann-Whitney tests were used to compare demographics, tumor characteristics and LOS. Multiple logistic and Cox regression analyses were used to compute Relative Risk (RR) for positive margins and mortality hazard ratios along with $95 \%$ Confidence Intervals (Cls), respectively.

Results: Of the 235 patients, 101 subjects had VATS while OT was performed in 134 patients. Age at diagnosis, sex, tobacco use, tumor location, and size was comparable for VATS and OT. No significant difference was observed in the RR of positive margins for VATS versus OT, $R R=0.56(95 \% \mathrm{Cl}=0.26,1.05)$. However, VATS had shorter median LOS compared to OT ( 4 vs. 6 days, respectively), $p=0.002$. A comparison of VATS versus OT showed no significant difference in the risk of recurrence, $\mathrm{HR}=1.21(95 \% \mathrm{Cl}=0.74,2.00)$, or death, $\mathrm{HR}=1.34(95 \%$ $\mathrm{Cl}=0.88,2.06)$, in the intent-to-treat population. Similarly, no significant differences in recurrence or mortality risk were observed between VATS versus OT for analyses conducted separately for each cancer stage group or those limited to patients with negative margins.

Conclusion: Our study indicates that compared to OT, VATS leads to shorter LOS while achieving comparable margins status, RFS and OS regardless of tumor stage at diagnosis and therefore can be considered as a favorable surgical approach in this group of patients.

Keywords: Video-assisted thoracic surgery; Open thoracotomy; Recurrence-free survival; Overall survival; Positive margins; Postoperative length of stay

Abbreviations: VATS: Video-Assisted Thoracoscopic Surgery; OT: Open Thoracotomy; RFS: Recurrence-Free Survival; OS: Overall Survival; LOS: Length of Stay; RR: Relative Risk; CC: Confidence Interval; DFS: Disease-Free Survival; IQR: Interquartile Rages

\section{Introduction}

Lung cancer is the leading cause of cancer-related mortalities around the world [1]. Potentially curable early stage Non-Small-Cell Lung Cancer (NSCLC) can be found in one-third of this patient population [2]. Surgical resection remains the backbone of treatment in resectable lung cancers. The introduction of VATS in 1994 [3] sparked interest in minimally invasive tumor resection. VATS has also been shown to have fewer postoperative complications [4] and has been associated with decreased postoperative pain and increased quality of life compared to OT [5]. Several studies have compared these two approaches indirectly, but no randomized controlled trial has investigated the long-term effect on outcomes. We aim to investigate the long-term Disease-Free Survival (DFS) and OS of patients with lung cancer undergoing lung resection by OT or VATS for resectable stage lung cancer.

\section{Patients and Methods}

\section{Surgical methods}

VATS lung resections were performed via a three-port incision technique including a 4-centimeter anterior axillary working port. The specimens were removed via the working port. Rib spreading was not required. A hilar dissection proceeding from anterior to posterior was performed for lobectomies. For OT resections, a standard posterolateral thoracotomy was used. Generally, bulky tumors, inability to tolerate one-lung ventilation, dense adhesions, en bloc chest wall resections, sleeve resections, neoadjuvant radiation therapy, or intraoperative

*Corresponding author: Salman Otoukesh, Department of Hematology/Oncology Loma Linda University Medical Center, Loma Linda, USA, Tel: +13106969932 E-mail: sotoukesh@llu.edu

Received October 14, 2018; Accepted October 22, 2018; Published October 29 2018

Citation: Shaheen S, Otoukesh S, Jabo B, Kaur M, Wheeler N, et al. (2018) Less is More: Video-Assisted Thoracic Surgery (VATS) vs. Open Thoracotomy (OT) in the Management of Resectable Lung Cancer. J Integr Oncol 7: 216. doi: 10.4172/23296771.1000216

Copyright: () 2018 Shaheen S, et al. This is an open-access article distributed under the terms of the Creative Commons Attribution License, which permits unrestricted use, distribution, and reproduction in any medium, provided the original author and source are credited. 
Citation: Shaheen S, Otoukesh S, Jabo B, Kaur M, Wheeler N, et al. (2018) Less is More: Video-Assisted Thoracic Surgery (VATS) vs. Open Thoracotomy (OT) in the Management of Resectable Lung Cancer. J Integr Oncol 7: 216. doi: 10.4172/2329-6771.1000216

Page 2 of 5

complications were reasons for selecting an OT approach or for requiring a conversion from VATS to OT.

\section{Study population}

Records for patients diagnosed with stage I through III resectable lung cancer treated at Loma Linda University Medical Center from May 2005 through May 2015 were retrieved through a retrospective chart review. Patients were subsequently divided into VATS and OT groups.

\section{Study outcomes}

The primary outcome of this study was RFS followed by OS as the secondary outcome. Survival was calculated from the date of surgery to the date of recurrence diagnosis/death or end of study follow-up.

\section{Study covariates}

Patient and tumor characteristics included age at diagnosis, sex, tobacco use, tumor location (Side and Lobe), stage, size and type of treatments including chemotherapy or radiotherapy.

\section{Statistical analyses}

Tumor and demographic characteristics were compared using Chi-square and Wilcoxon-Mann-Whitney tests. Purposeful variable selection approach was used to identify covariates that were included in the final models. A covariate-adjusted Cox proportional hazards model was used to compare RFS and OS between patients treated with VATS and those treated with OT. Profile likelihood was used to estimate $95 \%$ CIs. Proportionality was assessed using Shoenfeld residuals correlations and log-log survival plots. All tests were conducted using R software; $\mathrm{R}$ Core Team. R: A language and environment for statistical computing; $\mathrm{R}$ Foundation for Statistical Computing, Vienna, Austria.

\section{Results}

\section{Study population description}

From May 2005 through May 2015, 235 patients were diagnosed with stage I through III lung cancer. Of those, 134 and 101 patients received OT and VATS, respectively (Table 1). Median age at surgery (with Interquartile Rages, (IQR)) was similar for the two groups, OT $69(\mathrm{IQR}=61,77)$ and VATS $70(\mathrm{IQR}=63,76), \mathrm{p}=0.48$. Similarly, no difference in gender distribution was observed, $p=0.92$. Both VATS and OT had a higher proportion of tumors located in the right lung, 62 $(61.39 \%)$ and 78 (58.21\%) respectively, $\mathrm{p}=0.31$. Additionally, more than half of all tumors were located in the upper lobe with a slightly higher proportion seen for OT [78 (57.58\%)] than VATS [53 (52.48\%)], $\mathrm{p}=0.02$. In contrast, a higher proportion of stage I cancers was treated by VATS versus $\mathrm{OT}, \mathrm{p}=0.02$. There was no significant difference in median tumor size [3 $(\mathrm{IQR}=2,5)$ vs. $3(\mathrm{IQR}=2,4), \mathrm{p}=0.41]$ or percentage of tumors with negative margins [104 (89.66\%) vs. 89 (95.70\%), p=0.13] between OT and VATS, respectively. Compared to VATS, a higher proportion of OT patients received chemotherapy [38 (28.36\%) vs. $13(12.87 \%)$, $\mathrm{p}=0.02]$ and radiotherapy [30 (22.39\%) vs. $4(3.96 \%), \mathrm{p}<0.001]$ [6-17].

\section{Recurrence-free survival (RFS) and overall survival (OS)}

No significant differences in RFS (Figure 1$)(\mathrm{p}=0.23$ ) or OS (Figure 2) $(p=0.68)$ were observed between VATS versus OT in the KaplanMeier survival curves. After adjusting for covariates, the Cox regression models (Tables 2 and 3), show no difference in RFS, HR=1.26 $(95 \%$ $\mathrm{CI}=0.73,2.19)$, or OS, $\mathrm{HR}=1.34(95 \% \mathrm{CI}=0.85,2.10)$, between VATS and OT.

\begin{tabular}{|c|c|c|c|}
\hline Patients characteristics & $\begin{array}{l}\text { Open thoracotomy } \\
(n=134)\end{array}$ & VATS $(n=101)$ & p-value \\
\hline Age at diagnosis $\neq$ & $69(61-77)$ & $70(63-76)$ & 0.48 \\
\hline \multicolumn{3}{|l|}{ Sex } & 0.92 \\
\hline Male & $50(37.31 \%)$ & $37(36.63 \%)$ & \\
\hline Female & $84(62.69 \%)$ & $64(63.37 \%)$ & \\
\hline \multicolumn{3}{|l|}{ Side } & 0.31 \\
\hline Left & $53(39.55 \%)$ & $39(38.61 \%)$ & \\
\hline Right & $78(58.21 \%)$ & $62(61.39 \%)$ & \\
\hline Bilateral/unknown & $3(2.24 \%)$ & & \\
\hline \multicolumn{3}{|l|}{ Lobe } & 0.02 \\
\hline Lower & $33(25.00 \%)$ & $40(39.60 \%)$ & \\
\hline Middle & $10(7.58 \%)$ & $6(5.94 \%)$ & \\
\hline Upper & $76(57.58 \%)$ & $53(52.48 \%)$ & \\
\hline Bilateral/unknown & $13(9.85 \%)$ & $2(1.98 \%)$ & \\
\hline \multicolumn{3}{|l|}{ Stage $†$} & 0.02 \\
\hline 1 & $62(46.27 \%)$ & $66(65.35 \%)$ & \\
\hline 2 & $33(24.63 \%)$ & $20(19.80 \%)$ & \\
\hline 3 & $30(22.39 \%)$ & $13(12.87 \%)$ & \\
\hline Unknown & $9(6.72 \%)$ & $2(1.98 \%)$ & \\
\hline Size $\neq$ & $3(2-5)$ & $3(2-4)$ & 0.41 \\
\hline \multicolumn{3}{|l|}{ Margin status } & 0.13 \\
\hline Negative & $104(89.66 \%)$ & $89(95.70 \%)$ & \\
\hline Positive & $12(10.34 \%)$ & $4(4.30 \%)$ & \\
\hline Unknown & $18(13.43 \%)$ & $8(7.92 \%)$ & \\
\hline \multicolumn{3}{|l|}{ Chemotherapy } & 0.02 \\
\hline No & $85(63.43 \%)$ & 80 (79.21\%) & \\
\hline Yes & $38(28.36 \%)$ & $13(12.87 \%)$ & \\
\hline Unknown & $11(8.21 \%)$ & $8(7.92 \%)$ & \\
\hline \multicolumn{3}{|l|}{ Radiotherapy } & $<0.001$ \\
\hline No & $99(73.88 \%)$ & 97 (96.04\%) & \\
\hline Yes & $30(22.39 \%)$ & $4(3.96 \%)$ & \\
\hline Unknown & $5(3.73 \%)$ & & \\
\hline $\begin{array}{l}\text { Postoperative length of } \\
\text { stay }\end{array}$ & $6(4-7)$ & $4(3-6)$ & 0.002 \\
\hline
\end{tabular}

Table 1: Patient's characteristics by type of procedure.

\section{Length of stay (LOS)}

The median LOS was 2 days shorter among patients treated with VATS compared to those treated with OT $4(3,6)$ vs. $6(4,7), \mathrm{p}=(0.002)$ (Figure 3).

\section{Discussion}

VATS was performed initially in the 1990's. Since then there have been multiple studies advocating the superiority of VATS over conventional OT in terms of short and long-term side effects as well as hospital LOS [18-20]. However, some surgeons still prefer OT over VATS. In fact, according to the Society of Thoracic Surgeons General Thoracic Surgery Database, the percentage of VATS lobectomies performed in the United States are performed by VATS $[21,22]$ at high volume centers. One explanation for this may be due to the controversial results between several comparative studies in this field [23] since during the resectable years of its development, there was a lack of a clear definition of VATS between thoracic surgeons [24-26]. The goal of this study was to evaluate the outcomes in a low volume university setting over the last 10-year period, 2005-2015, where VATS was initiated in 2009 (Figure 4).

Our study, like other similar articles (Table 4), did not capture any 
Citation: Shaheen S, Otoukesh S, Jabo B, Kaur M, Wheeler N, et al. (2018) Less is More: Video-Assisted Thoracic Surgery (VATS) vs. Open Thoracotomy (OT) in the Management of Resectable Lung Cancer. J Integr Oncol 7: 216. doi: 10.4172/2329-6771.1000216

Page 3 of 5

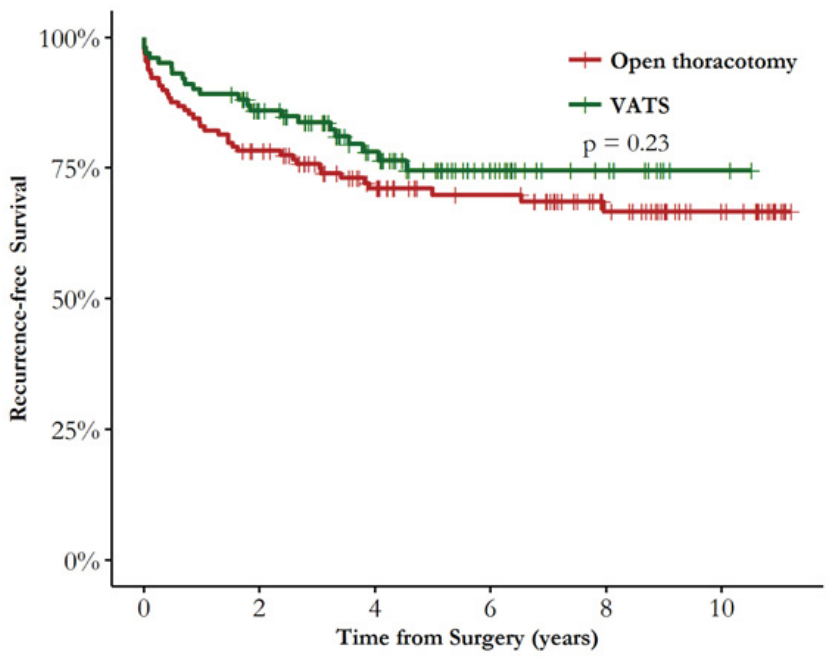

Number at Risk

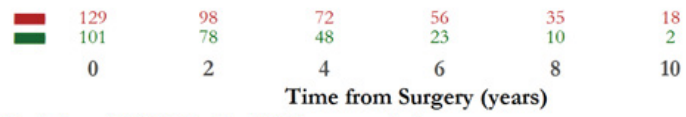

Abbreviations- VATS: Video Assisted Thoracoscopic Surgery

Figure 1: Kaplan-Meier survival curves for recurrence-free survival.

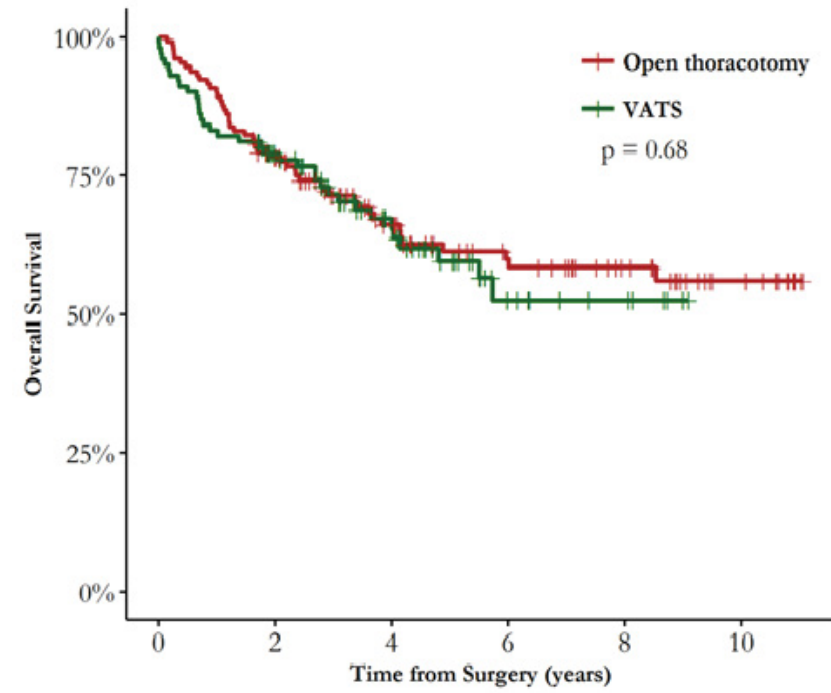

Number at Risk

\begin{tabular}{cccccc}
130 & 96 & 61 & 41 & 27 & 13 \\
101 & 69 & 38 & 12 & 6 & 0 \\
0 & 2 & 4 & 6 & 8 & 10 \\
& \multicolumn{5}{c}{ Time from Surgery (years) }
\end{tabular}

Abbreviations- VATS: Video Assisted Thoracoscopic Surgery

Figure 2: Kaplan-Meier survival curves for overall survival.

statistically significant findings between VATS and OT groups in terms of RFS and OS ( $\mathrm{p}=0.23$ and $\mathrm{p}=0.68$, respectively). Also similarly, VATS lobectomy was associated with shorter LOS and non-inferior long-term

\begin{tabular}{|l|l|}
\hline Patients characteristics & HR $\mathbf{( 9 5 \%} \mathbf{~ C l})$ \\
\hline Procedure & 1 \\
\hline Open thoracotomy & $1.26(0.73,2.19)$ \\
\hline VATS & $1.10(0.90,1.35)$ \\
\hline Age & \multicolumn{2}{|l|}{} \\
\hline Stage & 1 \\
\hline $0-1$ & $5.71(2.73,11.93)$ \\
\hline 2 & $9.81(4.87,19.78)$ \\
\hline 3 & $3.18(0.86,11.81)$ \\
\hline Unknown & \multicolumn{2}{|l|}{} \\
\hline Margin status & 1 \\
\hline Negative & $2.21(0.92,5.34)$ \\
\hline Positive & $2.21(1.12,4.38)$ \\
\hline Unknown &
\end{tabular}

Abbreviations-VATS: Video Assisted Thoracoscopic Surgery; HR: Hazard Ratio; Cl: Confidence Interval

Table 2: Covariate-Adjusted cox regression recurrence hazards ratios with $95 \%$ Confidence Interval $(\mathrm{Cl})$

\begin{tabular}{|l|l|}
\hline Patients characteristics & HR $\mathbf{( 9 5 \%}$ Cl) \\
\hline Procedure & 1 \\
\hline Open thoracotomy & $1.34(0.85,2.10)$ \\
\hline VATS & $1.36(1.11,1.68)$ \\
\hline Age & \multicolumn{2}{|l|}{} \\
\hline Stage & 1 \\
\hline $0-1$ & $1.82(1.03,3.19)$ \\
\hline 2 & $3.25(1.90,5.55)$ \\
\hline 3 & $3.21(1.30,7.89)$ \\
\hline Unknown & \multicolumn{2}{|l|}{} \\
\hline Margin status & 1 \\
\hline Negative & $2.12(1.04,4.32)$ \\
\hline Positive & $1.10(0.54,2.25)$ \\
\hline Unknown &
\end{tabular}

Abbreviations-VATS: Video Assisted Thoracoscopic Surgery; HR: Hazard Ratio; Cl: Confidence Interval

Table 3: Covariate-Adjusted cox regression recurrence hazards ratios with $95 \%$ Confidence Interval $(\mathrm{Cl})$.

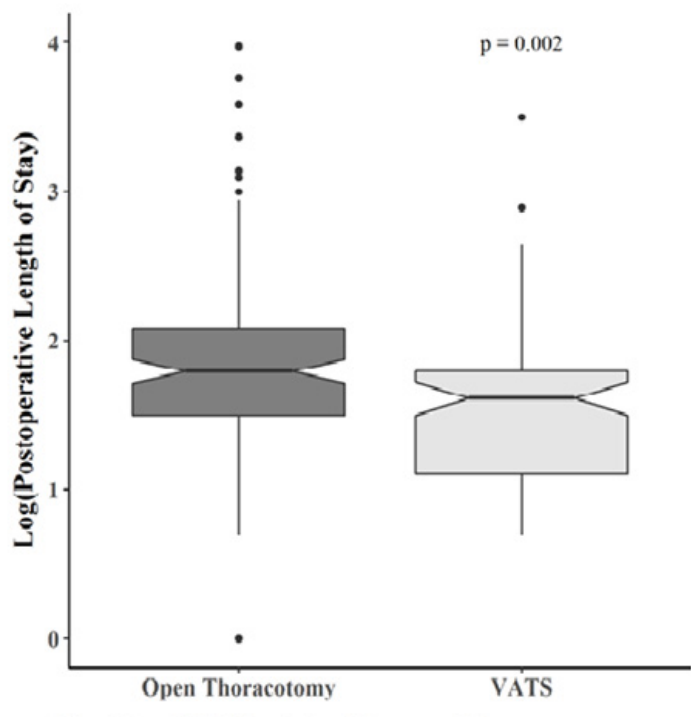

Abbreviations-VATS: Video Assisted Thoracoscopic Surgery

Figure 3: Postoperative length of stay by type of procedure. 
Citation: Shaheen S, Otoukesh S, Jabo B, Kaur M, Wheeler N, et al. (2018) Less is More: Video-Assisted Thoracic Surgery (VATS) vs. Open Thoracotomy (OT) in the Management of Resectable Lung Cancer. J Integr Oncol 7: 216. doi: 10.4172/2329-6771.1000216

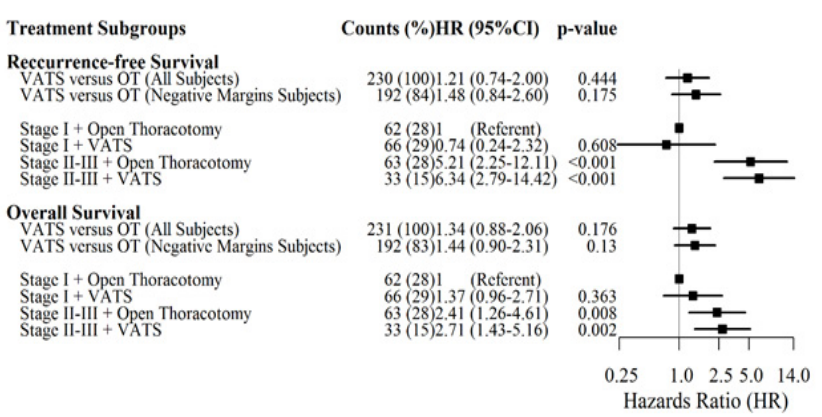

Abbreriations- VATS: Video Assisted Thoracoscopic Surgery; OT: Open Thoracotomy; HR: Hazard Ratio; CI: Confidence Interval

Figure 4: Forest plot for various treatment subgroups.

\begin{tabular}{|c|c|c|c|c|c|}
\hline $\mathrm{N}=$ Total Pts & $\begin{array}{l}\text { Shorter } \\
\text { LOS VATS } \\
\text { vs. OT }\end{array}$ & $\begin{array}{l}\text { LOS } \\
\text { (p-value) }\end{array}$ & $\begin{array}{l}\text { DFS } \\
\text { (p-value) }\end{array}$ & OS (p-value) & \begin{tabular}{|l} 
DFS/ \\
OS \\
(years)
\end{tabular} \\
\hline $\begin{array}{l}\mathrm{N}=160 \\
114 \text { VATS } \\
46 \text { OT }\end{array}$ & & & $\begin{array}{l}N \\
\text { difference } \\
p=0.27\end{array}$ & $\begin{array}{l}\text { No difference } \\
p=0.55\end{array}$ & 5 \\
\hline \begin{tabular}{|l|} 
Propensity \\
score \\
matching 88 \\
per group
\end{tabular} & $\begin{array}{l}\text { VATS } \\
2 \text { days }\end{array}$ & $p<0.05$ & $\begin{array}{l}N \\
\text { difference } \\
p=0.63\end{array}$ & $\begin{array}{l}\text { No difference } \\
p=0.27\end{array}$ & 3 \\
\hline $\begin{array}{l}\text { Propensity } \\
\text { score } \\
\text { matching } \\
175 \text { per } \\
\text { group }\end{array}$ & VATS & $p<0.05$ & $\begin{array}{l}N \\
\text { difference } \\
p=0.35\end{array}$ & $\begin{array}{l}\text { No } \quad \text { difference } \\
p=0.24\end{array}$ & 5 \\
\hline $\begin{array}{l}\mathrm{N}=55 \\
25 \text { VATS } \\
30 \text { OT }\end{array}$ & VATS & $p<0.05$ & & & \\
\hline $\begin{array}{l}\text { Propensity } \\
\text { score } \\
\text { matching } \\
1195 \text { per } \\
\text { group }\end{array}$ & & & $\begin{array}{l}N \\
\text { difference } \\
p=0.46\end{array}$ & $p=0.55$ & 3 \\
\hline $\begin{array}{l}80 \\
40 \text { VATS } \\
40 \text { OT }\end{array}$ & & & $\begin{array}{l}N \\
\text { difference } \\
p=0.44(I A) \\
p=0.48(I B)\end{array}$ & $\begin{array}{l}\text { No difference } \\
p=0.15(\mathrm{IA}) \\
p=0.20(\mathrm{IB})\end{array}$ & 5 \\
\hline $\begin{array}{l}\mathrm{N}=68350 \\
10554 \text { VATS } \\
57796 \text { OT }\end{array}$ & $\begin{array}{l}\text { VATS } \\
2 \text { days }\end{array}$ & $p<0.001$ & & & \\
\hline $\begin{array}{l}\mathrm{N}=389 \\
63 \text { VATS } \\
326 \text { OT }\end{array}$ & VATS & $p<0.001$ & & & \\
\hline $\begin{array}{l}\mathrm{N}=287 \\
98 \text { VATS } \\
189 \text { OT }\end{array}$ & VATS & $p<0.001$ & & & \\
\hline $\begin{array}{l}N=529 \\
156 \text { VATS } \\
373 \text { OT }\end{array}$ & $\begin{array}{l}\text { VATS } \\
2 \text { days }\end{array}$ & $p<0.05$ & $\begin{array}{l}N \\
\text { difference } \\
p=0.43\end{array}$ & $\begin{array}{l}\text { No difference } \\
p=0.76\end{array}$ & 3 \\
\hline $\begin{array}{l}\mathrm{N}=136 \\
74 \text { VATS } \\
62 \text { OT }\end{array}$ & VATS & $p<0.001$ & & & \\
\hline $\begin{array}{l}\mathrm{N}=741 \\
398 \text { VATS } \\
343 \text { OT }\end{array}$ & $\begin{array}{l}\text { VATS } \\
2 \text { days }\end{array}$ & $p<0.001$ & & $\begin{array}{l}\text { No difference } \\
p=0.12\end{array}$ & 5 \\
\hline
\end{tabular}

Abbreviations- LOS: Length Of Stay; Vats: Video Assisted Thoracoscopic Surgery: Ot: Open Thoracotomy; DFS: Disease-Free Survival; OS: Overall Survival

Table 4: Lobectomy associated with shorter LOS.

survival when compared with OT lobectomy. These results support previous findings from smaller single- and multi-institutional studies that suggest that VATS does not compromise oncologic outcomes when used for resectable stage lung cancer [27,28]. Over the last 15 years, there have been multiple studies, which have compared VATS to OT (Table 4). As noted in the table, these studies consistently showed decreased LOS and no difference in three to five-year disease free or OS. Our data is consistent with other data sets retrospectively comparing VATS and OT for resection of resectable non-small cell lung cancer $[10,28]$.

Although our current study did not capture any statistically significant value related to RFS, OS between two surgical modalities but compare to OT, in VATS lobectomy's incisions are smaller with no rib spreading which leads to have a faster recovery. Reviewing the previous studies showed although there was no difference in terms of the timing to receive adjuvant chemotherapy between VATS vs. OT [17] but patients who went under VATS had a better chance to tolerate and complete adjuvant chemotherapy courses, with less need for dose reduction and experiencing less related side effects [29] although still there is lack of data and results about any possible OS benefit [30-32].

Our study has several limitations. First and most importantly, our study is a single institution retrospective study. Specific information on patient selection criteria as well as differences in surgeons' experience is lacking and may have led to selection bias. VATS, like all newly developed minimally invasive surgical techniques, requires skills and experience in which not all surgeons have been trained.

\section{Conclusion}

Our study suggests that patients undergoing VATS lobectomy in a low-medium sized university setting have comparable long-term and short-term outcomes compared to national data in terms of DFS, OS, and shorter LOSs. This suggests referrals to high volume centers for lobectomy is not required as even low-medium volume centers with board-certified thoracic surgeons trained in VATS can achieve equivalent outcomes.

\section{References}

1. Verstegen NE, Oosterhuis JWA, Palma DA, Rodrigues G, Lagerwaard FJ, et al. (2013) Stage I-II non-small-cell lung cancer treated using either Stereotactic Ablative Radiotherapy (SABR) or lobectomy by Video-Assisted Thoracoscopic Surgery (VATS): outcomes of a propensity score-matched analysis. Ann Oncol 24: $1543-1548$.

2. Campbell DE, Greenberg RE, Polednak AP, Deshazer C, Bach PB, et al. (2000) Racial differences in the treatment of early-stage lung cancer. NEJM 342: 517-519.

3. McKenna RJ Jr (1994) Lobectomy by video-assisted thoracic surgery with mediastinal node sampling for lung cancer. J Thorac Cardiovasc Surg 107: 879-881.

4. Cao C, Manganas C, Ang SC, Peeceeyen S, Yan TD (2013) Video-Assisted thoracic surgery versus open thoracotomy for non-small cell lung cancer: a meta-analysis of propensity score-matched patients. Interact Cardiovasc Thorac Surg 16: 244-249.

5. Bendixen M, Jorgensen OD, Kronborg C, Andersen C, Licht PB (2016) Postoperative pain and quality of life after lobectomy via video-assisted thoracoscopic surgery or anterolateral thoracotomy for early stage lung cancer: a randomised controlled trial. The Lancet Oncology 17: 836-844.

6. Higuchi M, Yaginuma H, Yonechi A, Kanno R, Ohishi A, et al. (2014) Long-term outcomes after Video-Assisted Thoracic Surgery (VATS) lobectomy versus lobectomy via open thoracotomy for clinical stage ia non-small cell lung cancer. J Cardiothorac Surg 9: 88.

7. Jung HS, Kim HR, Choi SH, Kim YH, Kim DK, et al. (2015) Clinical feasibility and efficacy of Video-Assisted Thoracic Surgery (VATS) anatomical resection in patients with central lung cancer: a comparison with thoracotomy. J Thorac Dis 7: $1774-1779$.

8. Nwogu CE, D'Cunha J, Pang H, Gu L, Wang X, et al. (2015) VATS lobectomy 
Citation: Shaheen S, Otoukesh S, Jabo B, Kaur M, Wheeler N, et al. (2018) Less is More: Video-Assisted Thoracic Surgery (VATS) vs. Open Thoracotomy (OT) in the Management of Resectable Lung Cancer. J Integr Oncol 7: 216. doi: 10.4172/2329-6771.1000216

has better perioperative outcomes than open lobectomy: calgb 31001, an ancillary analysis of calgb 140202 (Alliance). Ann Thorac Surg 99: 399-405.

9. Erus S, Tanju S, Kapdagli M, Ozkan B, Dilege S, et al. (2014) The comparison of complication, pain, quality of life and performance after lung resections with thoracoscopy and axillary thoracotomy. Eur J Cardiothorac Surg 46: 614-619.

10. Paul S, Isaacs AJ, Treasure T, Altorki NK, Sedrakyan A (2014) Long term survival with thoracoscopic versus open lobectomy: propensity matched comparative analysis using seer-medicare database. BMJ 349: g5575.

11. Kuritzky AM, Ryder BA, Ng T (2013) Long-Term survival outcomes of videoassisted thoracic surgery (vats) lobectomy after transitioning from open lobectomy. Ann Surg Oncol 20: 2734-2740.

12. Paul S, Sedrakyan A, Chiu YL, Nasar A, Port JL, et al. (2013) Outcomes after lobectomy using thoracoscopy vs thoracotomy: a comparative effectiveness analysis utilizing the nationwide inpatient sample database. Eur $\mathrm{J}$ Cardiothorac Surg 43: 813-817.

13. Papiashvilli M, Stav D, Cyjon A, Haitov Z, Gofman V, et al. (2012) Lobectomy for non-small cell lung cancer: differences in morbidity and mortality between thoracotomy and thoracoscopy. Innovations (Phila) 7: 15-22.

14. Ramos R, Masuet C, Gossot D (2012) Lobectomy for early-stage lung carcinoma: A cost analysis of full thoracoscopy versus posterolateral thoracotomy. Surg Endosc 26: 431-437.

15. Park JS, Kim K, Choi MS, Chang SW, Han WS (2011) Video-Assisted Thoracic Surgery (VATS) lobectomy for pathologic stage I non-small cell lung cancer: a comparative study with thoracotomy lobectomy. Korean $\mathrm{J}$ Thorac Cardiovasc Surg 44: 32-38.

16. Scott WJ, Matteotti RS, Egleston BL, Oseni S, Flaherty JF (2010)A Comparison of perioperative outcomes of Video-Assisted Thoracic Surgical (VATS) lobectomy with open thoracotomy and lobectomy: results of an analysis using propensity score based weighting. Ann Surg Innov Res 4: 1.

17. D'Amico TA (2016) VATS lobectomy facilitates the delivery of adjuvan docetaxel-carboplatin chemotherapy in patients with non-small cell lung cancer. J Thorac Dis 8: 296.

18. Yim AP, Wan S, Lee TW, Arifi AA (2000) VATS lobectomy reduces cytokine responses compared with conventional surgery. Ann Thorac Surg 70: 243-247.

19. Muraoka M, Oka T, Akamine S, Tagawa T, Nakamura A, et al. (2006) Videoassisted thoracic surgery lobectomy reduces the morbidity after surgery for stage I non-small cell lung cancer. Jpn J Thorac Cardiovasc Surg 54: 49-55.

20. Whitson BA, Andrade RS, Boettcher A, Bardales R, Kratzke RA (2007) Video-assisted thoracoscopic surgery is more favorable than thoracotomy for resection of clinical stage I non-small cell lung cancer. Ann Thorac Surg 83: 1965-1970.
21. Park BJ (2012) Cost concerns for robotic thoracic surgery. Ann Cardiothorac Surg 1: 56-58.

22. Boffa DJ, Allen MS, Henning JD, Gaissert HA, Harpole DH, et al. (2008) Data from the Society of Thoracic Surgery General Thoracic Surgery database: the surgical management of primary lung tumors. J Thorac Cardiovasc Surg 135: 247-254.

23. Gopaldas RR, Bakaeen FG, Dao TK, Walsh GL, Swisher SG, et al. (2010) Video-assisted thoracoscopic versus open thoracotomy lobectomy in a cohort of 13,619 patients. Ann Thorac Surg 89: 1563-1570.

24. Swanson SJ, Herndon JE II, D'Amico TA, Demmy TL, McKenna RJ Jr. (2007) Video-assisted thoracic surgery lobectomy: report of CALGB 39802-a prospective, multi-institution feasibility study. J Clin Oncol 25: 4993-4997.

25. Yan TD, Black D, Bannon PG, McCaughan BC (2009) Systematic review and meta-analysis of randomized and nonrandomized trials on safety and efficacy of video-assisted thoracic surgery lobectomy for early-stage non-small-cell lung cancer. J Clin Oncol 27: 2553-2562.

26. Shigemura N, Akashi A, Funaki S, Nakagiri T, Inoue M, et al. (2006) Longterm outcomes after a variety of video-assisted thoracoscopic lobectomy approaches for clinical stage IA lung cancer: a multi-institutional study. J Thorac Cardiovasc Surg 132: 507-512.

27. Cattaneo SM, Park BJ, Wilton AS, Seshan VE, Bains MS (2008) Use of video-assisted thoracic surgery for lobectomy in the elderly results in fewer complications. Ann Thorac Surg 85: 231-235.

28. Yang CJ, Kumar A, Klapper JA, Hartwig MG, Tong BC, et al. (2017) A national analysis of long-term survival following thoracoscopic versus open lobectomy for stage I non-small-cell lung cancer. Ann Surg.

29. Jiang G, Yang F, Li X, Liu J, Li J, et al. (2011) Video-Assisted Thoracoscopic Surgery (VATS) is more favorable than thoracotomy for administration of adjuvant chemotherapy after lobectomy for non-small cell lung cancer. World J Surg Oncol 9: 170.

30. Petersen RP, Pham D, Burfeind WR, Hanish SI, Toloza EM, et al. (2007) Thoracoscopic lobectomy facilitates the delivery of chemotherapy after resection for lung cancer. Ann Thorac Surg 83: 1245-1249.

31. Cao C, Manganas C, Ang SC, Yan TD (2012) A systematic review and metaanalysis on pulmonary resections by robotic video-assisted thoracic surgery Ann Cardiothorac Surg 1: 3-10.

32. Yan TD, Black D, Bannon PG (2009) Systematic review and meta-analysis of randomized and nonrandomized trials on safety and efficacy of video-assisted thoracic surgery lobectomy for early-stage non-small-cell lung cancer. J Clin Oncol 27: 2553-2562 